\title{
Visual search asymmetry depends on target-distractor feature similarity: Is the asymmetry simply a result of distractor rejection speed?
}

\author{
Yichi (Raven) Zhang ${ }^{1,2} \cdot$ Serge Onyper ${ }^{1}$ \\ Published online: 29 July 2019 \\ (C) The Psychonomic Society, Inc. 2019
}

\begin{abstract}
Previous studies have shown that in visual search, varying the target and distractor familiarity produces a search asymmetry: Detecting a novel target among familiar distractors is more efficient than detecting a familiar target among novel distractors. One explanation is that novel targets have enhanced salience and are detected preattentively. Conversely, familiar distractors may be easier to reject. The current study postulates that target-distractor feature similarity, in addition to target or distractor familiarity, is a key determinant of visual search efficiency. The results of two experiments reveal that visual search is more efficient when distractors are familiar regardless of target familiarity, but only when the target-distractor similarity is high. When similarity is low, the visual search asymmetry disappears and the search times become highly efficient, with search slopes not different from zero regardless of target or distractor familiarity. However, although distractor familiarity plays an important role in inducing the search asymmetry, comparisons of search efficiency in target-present and target-absent trials reveal that search asymmetries cannot be explained solely by the faster speed of rejecting familiar distractors, as proposed by previous studies. Rather, distractor familiarity influences processes outside of stimulus selection, such as search monitoring and termination decisions. Competition among bottom-up item salience effects and top-down shape recognition processes is proposed to account for these findings.
\end{abstract}

Keywords Visual search $\cdot$ Asymmetry $\cdot$ Familiarity $\cdot$ Similarity $\cdot$ Target $\cdot$ Distractor $\cdot$ Feature

Our everyday life is full of tasks that require visual search, from avoiding obstacles as we drive to our office or hike an unfamiliar trail, to locating keys, identifying new e-mail messages, or recognizing our child in a crowd of other children. The ability of stimuli in the environment to capture visual attention and the limited capacity of the human attentional processes have been the focus of considerable study in the past 40 years (e.g., Buetti, Cronin, Madison, Wang, \&

Serge Onyper

sonyper@stlawu.edu

Yichi (Raven) Zhang

yzhang96@umd.edu

1 Department of Psychology, St. Lawrence University, Canton, NY 13617, USA

2 Present address: University of Maryland, College Park, MD, USA
Lleras, 2016; Duncan \& Humphreys, 1989; Rosenholtz, Huang, Raj, Balas, \& Ilie, 2012; Treisman \& Gelade, 1980; Wolfe, 2001). One question that received significant research interest concerns the characteristics of stimuli that facilitate or diminish the efficiency of attentional capture. The phenomenon of visual search asymmetries offers a fertile means to study this question. Early investigations found that searching for a target that possesses an added basic feature not shared with distractors (e.g., a circle with a lower half bisected by a vertical line among a variable number of complete circles) resulted in flatter reaction time slopes than when the distractors possessed an added feature not shared with the target (e.g., a complete circle; Treisman \& Souther, 1985). This search asymmetry results from a discrepancy in the number of basic features in the target-distractor pairs: The added feature of the target serves as a preattentive cue that automatically captures attention regardless of the number of singlefeature distractors, resulting in a highly efficient search where such target "pops out." Locating a single-feature target among distractors that combine multiple features, on the other hand, necessitates the more demanding serial search where the speed 
of the search increases with the number of distractors (inefficient search; Wolfe, 1998).

The claim that searching for items with a greater number of basic features than accompanying distractors is more efficient than searching for items with fewer features has generated considerable research on what qualifies as a basic feature. Several studies have reported that the target and distractor familiarity can produce an asymmetry, whereby searching for a novel target among familiar distractors is more efficient than searching for a familiar target among novel distractors (Malinowski \& Hübner, 2001; Rauschenberger \& Chu, 2006; Reicher, Snyder, \& Richards, 1976; Shen \& Reingold, 2001; Wang, Cavanagh, \& Green, 1994; Wang, Zhang, He, \& Jiang, 2010; Wolfe, 2001). Because visual search can potentially be affected both by the familiarity of the critical, sought-after information, and by the background, irrelevant or to-beignored stimuli, understanding the role of familiarity is key to understanding how visual search performance, whether in the laboratory or applied settings, can be enhanced.

To explain why varying the target-distractor familiarity can introduce a search asymmetry, some researchers have held that the absence of familiarity - that is, novelty itself - can be regarded as one kind of basic feature that can capture attention in parallel processing (Treisman \& Gormican, 1988; Wang et al., 1994). For instance, in an experiment that manipulated target and distractor novelty factorially, only the novel-target/ familiar-distractors condition resulted in a nearly flat slopes of $1.5 \mathrm{~ms} /$ item (evidence of a highly efficient search); the slope exceeded $30 \mathrm{~ms} /$ item in all other conditions (evidence of an inefficient search; Wang et al., 1994). Based on these results, Wang and colleagues argued that to make visual search efficient, there must be a difference in familiarity between the target and the distractors (see also Duncan \& Humphreys, 1989). When both are familiar (or novel), attention is equally distributed across the target and the distractors and the target cannot be quickly spotted. When the target is familiar and distractors novel, the distractors capture attention and must be processed first, which results in a slow, inefficient search. Therefore, only when the target is novel while the distractors are familiar is the search efficient because the target item triggers preattentive processing and thus can be quickly identified (see also Treisman \& Gormican, 1988; Treisman \& Souther, 1985).

In contrast to the "target orienting" hypothesis proposed by Wang et al. (1994), the search asymmetry may result from a difference in the speed of rejecting distractors (Malinowski \& Hübner, 2001; Shen \& Reingold, 2001; Wolfe, 2001). According to the "distractor processing" view, in the search of a novel target among familiar distractors, the search is more serial and less parallel than what the preattentive theory would predict. Novelty does not serve as a preattentive feature that can be immediately captured and processed. Instead, a novel target is more quickly identified among familiar distractors because familiar distractors can be more easily rejected during the search for the target, whereas it takes longer for novel distractors to be rejected.

If this alternative explanation is correct, then searching for a familiar target among familiar distractors should be as efficient as searching for a novel target among familiar distractors, since familiarity of the target item is less important if response time is mainly determined by the speed of rejecting distractors. Although this prediction was not borne out by the results of Wang and others (1994), who found longer search times for a familiar compared with a novel target among familiar distractors, it is consistent with other findings. In an experiment conducted by Malinowski and Hübner (2001), German speakers who had no previous experience with the Cyrillic alphabet and Slavic speakers were tested on a search task involving the letter $N$ and the mirror $N$. While the Slavic speakers were familiar with both letters, the German speakers were only familiar with $N$. The German speakers exhibited a search asymmetry: Searching for the mirror $N$ among the $N$ distractors was more efficient $(1 \mathrm{~ms} /$ item $)$ than searching for the $N$ among the mirror $N$ distractors ( $38 \mathrm{~ms} /$ item). However, the Slavic speakers produced flat slopes in all conditions (3 $\mathrm{ms} /$ item when the distractor was $N ; 0.3 \mathrm{~ms} /$ item when the distractor was a mirror $N$ ). For the Slavic speakers, searching for a familiar target among familiar distractors was also efficient.

In a similar experiment conducted by Shen and Reingold (2001), native Chinese speakers participated in a visual search task using valid Chinese characters and nonsensical characters as targets and distractors. Consistent with Malinowski and Hübner's (2001) findings, the search was efficient when the distractors were familiar regardless of the familiarity of the target. When the distractors were novel, the search was much less efficient. Using $V$ or inverted $V$ as the target and $A$ or inverted $A$ as the distractors, Wolfe (2001) reported similar results: Searching among the familiar distractors elicited flatter slopes than searching among novel distractors regardless of target familiarity. These results thus suggest, contrary to the target-orienting hypothesis, that distractor familiarity, rather than target novelty, drives search asymmetries.

The prima facie discrepancy in the findings regarding whether looking for a familiar target among familiar distractors is as efficient as looking for a novel target among familiar distractors may be due to a potential confound: The orthographic similarity between the target and the distractor, which decreases the efficiency of the search (Duncan \& Humphreys, 1989; Palmer, Verghese, \& Pavel, 2000; see also Buetti et al., 2016). Orthographic (feature) similarity is a key determinant of demands on resources consumed during the focused attention stage of the visual search (Duncan \& Humphreys, 1989). For instance, in Wang et al. (1994), the targets (5 or 2$)$ and the distractors ( 2 or 5 ) in the familiartarget/familiar-distractors condition only differed in terms of 
the position of the vertical lines, whereas in the novel-target/ familiar-distractors condition, the target $(N$ or $Z)$ and the distractors (mirror $N$ or mirror $Z$ ) differed in terms of the direction of the diagonal line (see Table 1). It has been suggested that orientation is a basic feature detected preattentively (Wolfe, 2001), and thus a difference in the orientation of diagonals may be more salient than a difference in the positioning of the verticals.

Moreover, configuring features in a particular way can increase the difficulty of the search: For instance, asking observers to locate $T \mathrm{~s}$ in random orientation among $L \mathrm{~s}$ in random orientation is more difficult than searching for the same stimuli in proper orientation (Wolfe, Cave, \& Franzel, 1989; see also Shen \& Reingold, 2001, for a similar conclusion). Importantly, with respect to the features shared, changing the position of the vertical lines does not affect the number of features shared between 2 and 5 , whereas altering the direction of the slanted line (e.g., $N$ and mirrored $N$ ) decreases the number of shared features. Thus, the difference in features used in the two conditions - configuration of vertical lines versus direction of diagonal lines - may have affected the results of Wang and colleagues.

In order to minimize the influence of orthographic basic features, the similarity between the target and the distractor must be held constant across different experimental conditions. This problem was to some extent addressed by Shen and Reingold (2001), who rotated the Chinese character “古” to create the four familiar/novel experimental conditions using the following characters: “古”, “叶”, inverted “古”, and mirror “叶” (see Table 1). For Chinese speakers, “古” and “叶” are legitimate Chinese characters (meaning "old" and "leaf," respectively) and are thus familiar, whereas the inverted “古” and the mirror "叶" are novel, nonsensical characters. Their results for respondents familiar with Chinese characters showed that while the two familiar-distractors conditions produced flatter slopes than the two novel-distractors conditions, the slopes in the novel-target/familiar-distractors and familiartarget/familiar-distractors conditions did not significantly differ from each other, which in turn supported the view that distractor familiarity is the key determinant of the visual search asymmetry (see also Horstmann, 2009; Horstmann, Becker, Bergmann, \& Burghaus, 2010).

The majority of the findings reviewed thus far suggest that distractor rejection speed is the cause of the familiarity-driven search asymmetry, while the characteristics of the target matter little, if at all. However, previous findings have not ruled out other ways in which distractor familiarity can cause the asymmetry. As a result, in the current series of experiments we hope to evaluate several possibilities regarding the role of familiarity in influencing the search function. These possibilities are diagrammed in Fig. 1, which focuses on distractor, rather than target, familiarity as the driving factor behind the asymmetry, and assumes that the search requires the resources of the capacity-limited attentive (rather than preattentive) processing, in the language of the feature-integration theory of attentional selection (Treisman \& Gelade, 1980). Both the slopes and intercepts for target-present and target-absent trials must be examined to evaluate the role of distractor processing. Figure 1a is consistent with the view that distractor familiarity is the critical factor to affect the slope of the search function, with novel distractors producing steeper slopes than familiar distractors both for target-present and target-absent trials, because novelty of distractors affects the item selection (attentive) stage of processing. This view aligns with the findings of Malinowski and Hübner (2001), Mruczek and Sheinberg (2005), and Shen and Reingold (2001).

One can also envision a situation where the effects of distractor familiarity on the search function lie outside of the item selection stage. These effects can be preattentive (e.g., distractor novelty may add a cost before an item-by-item search is executed) or postattentive (e.g., distractor novelty may affect search termination or other response criteria). Such possibility is sketched in Fig. 1b. In this case, distractor novelty effects are additive with the effects of set size: A fixed cost not related to the item selection stage is added to the intercept of the search function. The slopes, on the other hand, do not vary as a function of distractor familiarity. While it is not necessary for the familiarity of distractors to influence search RTs for target-absent trials to the same degree of magnitude as RTs for target-present trials, because the search is expected to be more exhaustive when no target can be found, it is reasonable to predict that the intercept differences for target-absent trials will be larger.

Figure 1c sketches a scenario where distractor familiarity does not affect the slope of the search function: The slopes for target-absent trials for novel and familiar distractors are similar, suggesting that the rejection rate for the two types of distractors does not vary differentially with set size. Any

Table 1 Representative stimuli from Wang et al. (1994) and from Experiment 4 of Shen and Reingold (2001)

\begin{tabular}{|c|c|c|c|c|}
\hline & \multicolumn{4}{|c|}{ Experimental Conditions } \\
\hline & $\begin{array}{l}\text { Familiar-target/ } \\
\text { Familiar-distractors }\end{array}$ & $\begin{array}{l}\text { Unfamiliar-target/ } \\
\text { unfamiliar-distractors }\end{array}$ & $\begin{array}{l}\text { Familiar-target/ } \\
\text { unfamiliar-distractors }\end{array}$ & $\begin{array}{l}\text { Unfamiliar-target/ } \\
\text { familiar-distractors }\end{array}$ \\
\hline Wang et al. (1994) & 与 / 己 & பா / ப & $N / n$ & $n / N$ \\
\hline $\begin{array}{l}\text { Shen and Reingold } \\
\text { (2001; Exp. 4) }\end{array}$ & 古 / 마 & 무 / 吅 & 古 / 吅 & 무 / 叶 \\
\hline
\end{tabular}




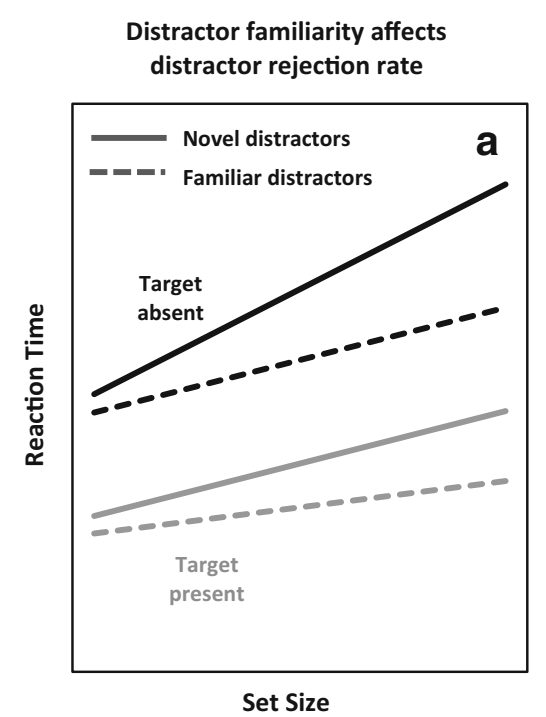

Fig. 1 Predicted search functions when distractor novelty/familiarity affects the efficiency of item selection during a capacity-limited attentive search captured by the distractor rejection rate (a). The search functions shown in Panel $\mathrm{b}$ are consistent with the notion that distractor familiarity affects processes outside of item selection, which is reflected in the
Distractor familiarity does not affect distractor rejection rate

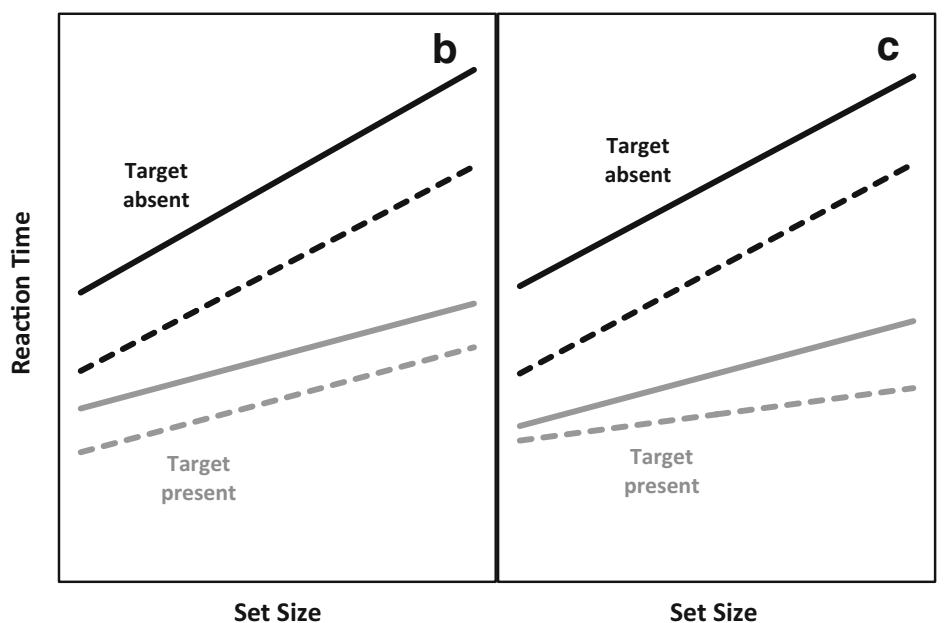

intercept differences between novel and familiar distractors. Panel c sketches a scenario in which the differences in the rejection speed of novel and familiar distractors are insufficient to account for the costs of distractor novelty.

distractors remain constant. In addition, the total number of features in previous studies has typically been held constant across target-distractor pairs. However, if similarity of basic features between the target and the distractor modulates the presence or the magnitude of the visual search asymmetry, then it is important to manipulate its effects directly, which is what the current study aims to do.

There are other reasons to examine the role of targetdistractor similarity more closely: For instance, Shen and Reingold (2001) found that when distractors and targets differed in the presence of a salient low-level feature, the search asymmetry was magnified due to the facilitation of the search in the novel-target familiar-distractors condition. In other words, familiarity-based stimulus detection mechanisms can be strengthened or weakened in the presence of higher or lower target-distractor similarity. The reasons for this interaction are unclear, however: Does similarity affect distractor rejection speed, or does it affect processes outside of the item selection stage? For instance, recent work suggests that targetdistractor similarity can influence preattentive processing (Buetti et al., 2016). Moreover, Shen and Reingold's conclusion itself has yet to be replicated. Broadly speaking, the causes of variability in slopes and intercepts sketched in the patterns of Fig. 1 can be extended to situations where orthographic similarity varies between targets and distractors.

In the current study, we attempted to extend Shen and Reingold's (2001) results in English speakers by systematically controlling the target-distractor similarity via confusion matrices that help define features essential to letter or character recognition (Fiset et al., 2009). Such features, for instance, 
include line intersections or terminations and line horizontals or verticals. These confusion matrices are typically developed by asking observers to discriminate among letters or features under demanding conditions, such as very low contrast, or by masking information hypothesized to be critical for stimulus identification. This approach minimizes the possibility that the stimulus set used in the visual search task is subject to an inherent asymmetry, which is a potential confound few previous studies have controlled (Rosenholtz, 2001). Moreover, by generating a large number of target-distractor pairs, we can reduce the potential effect of target experience on search efficiency (Awh, Belopolsky, \& Theeuwes, 2012; Hout \& Goldinger, 2012; Mruczek \& Sheinberg, 2005).

Careful control of the degree of target-distractor similarity will furthermore enable us to explain the variability in response-time slopes obtained by searching for a target among familiar distractors. In previous work, such slopes have ranged from highly efficient (well below the 10 -ms per item criterion often used to infer preattentive target identification) to inefficient (at or above 25-35-ms per item, an indicator of limitedcapacity attentive search; for a brief review, see Wolfe, 2001). Given that the question of whether or not novelty is a feature that can be detected preattentively has been explored but not resolved in earlier work (e.g., Malinowski \& Hübner, 2001; Shen \& Reingold, 2001; Wang et al., 1994; Wolfe, 2001), such controls can yield productive insights into the nature of basic features and attentional capture more generally.

To isolate the search effects due to stimulus familiarity from the influence due to other top-down, memory-driven factors such as observer experience and expectations, we used a singleton (odd item) search task. A notable feature of previous studies that considered the role of familiarity in producing search asymmetries is the reliance on tasks with a known target identity. In such tasks, attentional control is a function of voluntary stimulus selection properties governed by the current goals of the observer. A singleton search should limit the influence of such top-down mechanisms, particularly when target and distractor stimuli are drawn from a large population of exemplars. Importantly, results from a singleton search task may allow us to differentiate between theoretical accounts that postulate different degrees of importance of prior knowledge of the object/target in producing a search asymmetry. For instance, some accounts suggest that such knowledge is necessary (e.g., Navalpakkam \& Itti, 2005; Wolfe, 1994), while others either purport that bottom-up, saliencydriven mechanisms can provide sufficient explanation (e.g., Rosenholtz, 2001; Saiki, 2008) or make no explicit predictions regarding the role of the top-down influences (for a brief review, see Saiki, Koike, Takahashi, \& Inoue, 2005). While the role of target-distractor familiarity has not yet been examined with a singleton search, evidence suggests that search asymmetries can be obtained even when observers execute a search without a predefined target (Saiki et al., 2005).
We examined the role of target and distractor familiarity in two experiments. Experiment 1 used a 3 (target-distractor familiarity: familiar-target/familiar-distractor, familiar-target/ novel-distractor, and novel-target/familiar-distractor) $\times 2$ (target-distractor similarity: high and low) $\times 3$ (set size: 4,10 , and 16 items) repeated-measures design. It employed a large visual field and presented stimuli in completely random locations. In Experiment 2, a smaller visual field was used with the stimuli in Set Sizes 2 through 8 arranged along a concentric circle.

If the visual search asymmetry results solely from a difference in the speed of rejecting familiar versus novel distractors, then we would expect that both the familiar-target/familiardistractors and the novel-target/familiar-distractors conditions elicit a more efficient search than the familiar-target/noveldistractors condition, regardless of the presence of a target (e.g., Fig. 1a). By contrast, if target novelty exerts a pop-out effect driven by target saliency, the novel-target/familiardistractors condition should be more efficient than the two familiar target conditions. Our predictions, however, are that the degree of feature overlap between the target and the distractors will modulate the familiarity-induced asymmetry effects: That is, target novelty or distractor familiarity may become more critical to successful target identification with increased orthographic overlap between targets and distractors. These effects may not necessarily be found in the capacity-limited item-selection processing stage or may affect the item selection stage without affecting distractor rejection rates (see Fig. 1b-c).

\section{Experiment 1}

\section{Method}

Participants Fifty-five undergraduate students (ages 18-22 years) completed the study in exchange for $\$ 10$ or course credit. Participants reported normal or corrected-to-normal vision. Eight participants were excluded from analysis because they failed to meet the $83 \%$ response accuracy criterion.

Stimuli Stimuli consisted of 12 uppercase letters and 24 nonsensical characters (see Table 2). Each uppercase letter was paired with two nonsensical characters. The confusion matrix (Fiset et al., 2009; Geyer \& DeWald, 1973) provided the basis for determining similarity. The matrix identified 10 features shared by the capital English letters, such as line intersections, line terminations, verticals/horizontals, right and left slants, and curves. Uppercase letters $C, G, E, F, P, R$, and their corresponding nonsensical characters formed the high-similarity group: These letter/character pairs shared at least $67 \%$ of features. Letters $O, Z, U, B, T, D$, and their corresponding nonsensical characters formed the low-similarity group: These pairs shared fewer than $27 \%$ of features. For example, $\mathrm{P}$ 
Table 2 Target-distractor pairs used in Experiment 1 and Experiment 2

Experimental condition

\begin{tabular}{|c|c|c|c|c|c|c|c|c|c|c|c|}
\hline \multicolumn{4}{|c|}{ Familiar target/familiar distractor } & \multicolumn{4}{|c|}{ Familiar target/novel distractor } & \multicolumn{4}{|c|}{ Novel target/familiar distractor } \\
\hline \multicolumn{2}{|c|}{ High similarity } & \multicolumn{2}{|c|}{ Low similarity } & \multicolumn{2}{|c|}{ High similarity } & \multicolumn{2}{|c|}{ Low similarity } & \multicolumn{2}{|c|}{ High similarity } & \multicolumn{2}{|c|}{ Low similarity } \\
\hline CG & GC & OZ & $\mathrm{ZO}$ & $\mathrm{CC}$ & $\mathrm{C}^{\prime}$ & O० & 06 & $\mathrm{CC}$ & ${ }^{\circ} \mathrm{C}$ & $\mathrm{OO}$ & 60 \\
\hline $\mathrm{EF}$ & $\mathrm{FE}$ & UB & BU & $\mathrm{Gg}$ & G1 & $\mathrm{Zz}$ & $\mathrm{Z} 7$ & $g G$ & $1 G$ & $\mathrm{zZ}$ & 72 \\
\hline \multirow[t]{4}{*}{ PR } & $\mathrm{RP}$ & $\mathrm{TD}$ & $\mathrm{DT}$ & $\mathrm{Ee}$ & E2 & $\mathrm{Uu}$ & U8 & $\mathrm{eE}$ & $2 \mathrm{E}$ & $u U$ & $8 \mathrm{U}$ \\
\hline & & & & $\mathrm{Ff}$ & F 3 & $\mathrm{Bb}$ & B9 & $\mathrm{fF}$ & $3 \mathrm{~F}$ & $\mathrm{bB}$ & $9 \mathrm{~B}$ \\
\hline & & & & $\mathrm{Pp}$ & P4 & Tt & TO & $\mathrm{pP}$ & $4 \mathrm{P}$ & tT & OT \\
\hline & & & & $\mathrm{Rr}$ & R5 & $\mathrm{Dd}$ & $D^{-}$ & $r R$ & $5 R$ & $\mathrm{dD}$ & $-D$ \\
\hline
\end{tabular}

would include two intersections, one line termination (bottom), a vertical, two horizontals, and a curve open to the left, whereas a nonsensical character similar to it, $\mathrm{P}$, would include an additional intersection, termination, and a vertical, thus sharing 70\% (7 out of 10) features with P. Similarly, "4," which is the other nonsensical character paired with $P$, included two additional intersections and a vertical, thereby also sharing $70 \%$ features with $\mathrm{P}$. The cutoff $-67 \%$ for the highsimilarity conditions and $27 \%$ for the low-similarity conditions - was chosen arbitrarily in order to generate an ample number of stimuli while ensuring a large enough difference in shared features between the two similarity conditions. All letters and nonsensical characters were generated based on a $4 \times 5$ matrix using font creation software. As shown in Table 2, we created six conditions by crossing target-distractor familiarity (three levels) with similarity between targets and distractors (two levels).

Procedure Stimuli were presented using Inquisit Lab 4. They were displayed on 23-inch monitors running at 1,920 × 1,080 resolution with $60-\mathrm{Hz}$ refresh rate. Stimuli subtended 25 degrees of visual angle horizontally and 12 degrees vertically, with participants positioned approximately $68 \mathrm{~cm}$ away from the screen. Each trial began with a $300-\mathrm{ms}$ presentation of a central fixation point ("+"), followed by the stimulus display, with a 1,000-ms intertrial interval. Stimuli were presented in uppercase font; the Height $\times$ Width of each character was $0.42^{\circ} \times 0.33^{\circ}$. Participants were required to determine, as quickly as possible, whether the display contained a single item (referred to as target) that was different from all other items. As a result, task instructions differed from the conventional visual search task instructions where the participant must decide whether a particular known target is present or absent to accommodate the fact that the target item is not known ahead of time and that it varies from trial to trial. While there is evidence to suggest that varying task instructions can affect response latency patterns (e.g., Lee, Sobel, York, \& Puri, 2018), as we discussed in the introduction, the absence of a predefined target does not have a significant effect on the search asymmetry (Saiki et al., 2005). On each trial, participants were instructed to "press the ' $F$ ' key for target-present or press the ' $\mathrm{J}$ ' key for target-absent displays." These instructions were displayed at the bottom of the screen.

The experiment comprised a practice phase and a test phase. During practice, participants completed 10 trials with letter pairs randomly selected from the familiar target/familiar distractor high-similarity condition; they were alerted if they made an incorrect response after each trial. The test phase consisted of 10 blocks of 36 trials. Within each block, trials varied in target-distractor familiarity (familiar-target/noveldistractor, novel-target/familiar-distractor, and familiar-target/ novel-distractor), target-distractor similarity (high vs. low), set size (4, 10, and 16 items), and type (target present vs. target absent; see Fig. 2 for a representation of sample trials at Set Size 10). Thus each target-distractor combination was presented 10 times over the course of the experiment; a given letter/character appeared equally often as a target and as a distractor, ensuring that the targets were not more distinguishable than the distractors. The distractors on each trial were homogeneous. Figure 2 provides examples of trials in each experimental condition. Items were shown in random order within each block, and blocks with accuracy below $83 \%$ (i.e., 30 trials out of 36 in a block of trials) were repeated. To ensure sufficiently high accuracy of responding, accuracy feedback was provided after each block wherein accuracy dropped below 83\%, and participants were prompted to increase their accuracy.

The experiment lasted 30-45 minutes, depending on the rate of accuracy maintained by the participant. Participants who could not achieve $83 \%$ accuracy overall were dismissed from the study.

\section{Results}

Response times and error rates The dependent variables of median response times (RTs) for correct responses in each condition and error rates were subjected to repeated- 


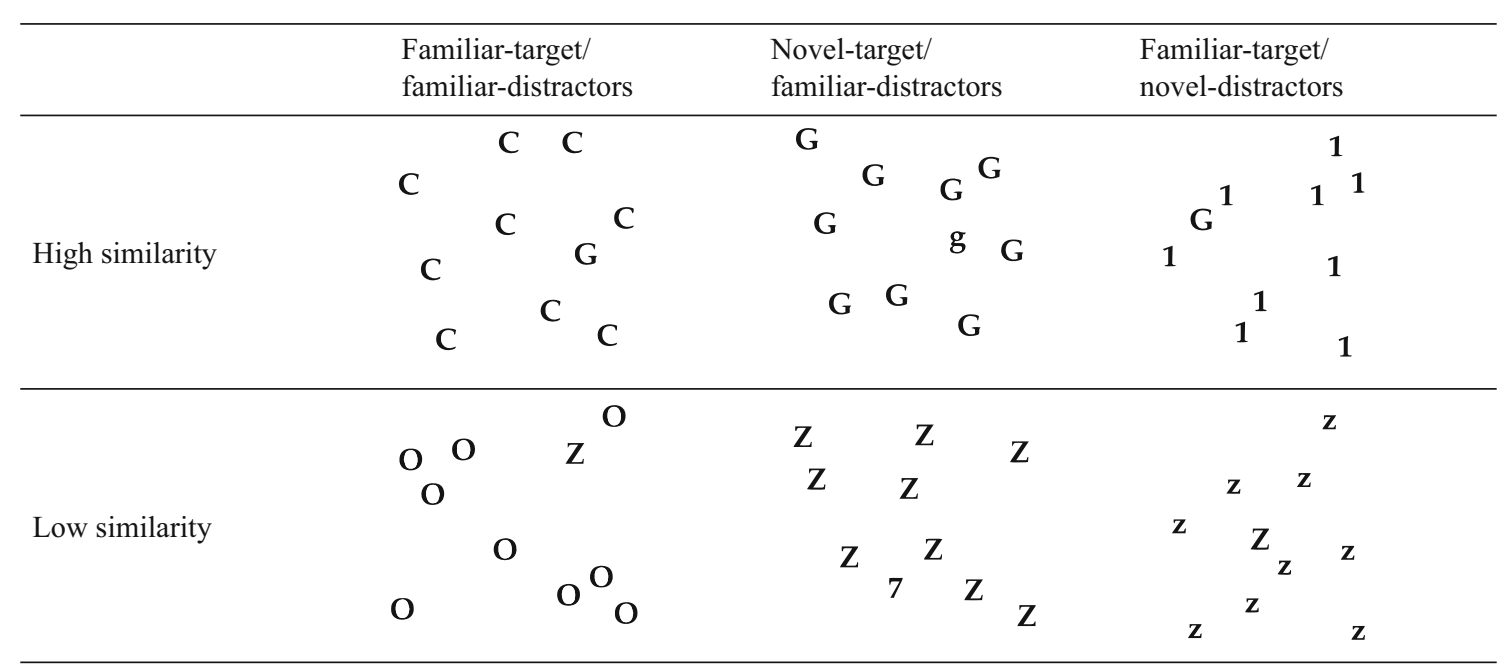

Fig. 2 Representative trials from Experiment 1 (Set Size 10). The search for a singleton target is parallel and equally efficient in all conditions when target-distractor similarity is low. However, when similarity is high,

measures analysis of variance (ANOVA) with Bonferroniadjusted pairwise comparisons. Figure 3 displays mean RTs and error rates as a function of target presence, similarity condition, familiarity condition, and set size. For target-present trials, a three-way repeated-measures ANOVA confirmed slower RTs when similarity was high, $F(1,46)=357.97, p<$ $.001, \eta_{\mathrm{p}}{ }^{2}=.89$, and the distractors novel, $F(2,92)=11.87, p<$ $.001, \eta_{\mathrm{p}}^{2}=.21$, a finding qualified by a significant similarity by familiarity interaction, $F(2,92)=11.94, p<.001, \eta_{\mathrm{p}}{ }^{2}=.21$. Follow-up analyses revealed that when the target-distractor similarity was low, the effect of target-distractor familiarity was not statistically significant. When similarity was high, however, search times for the novel-distractors condition were significantly longer than for either of the familiar-distractors conditions $(p s<.001)$, which themselves did not differ. The three-way ANOVA further revealed that larger set sizes led to higher RTs, $F(2,92)=42.93, p<.001, \eta_{\mathrm{p}}{ }^{2}=.48$, disproportionately so for highly similar target-distractor pairs, $F(2,92)$ $=24.18, p<.001, \eta_{\mathrm{p}}{ }^{2}=.35$. Follow-up comparisons indicated that for highly similar pairs, the RTs for the three familiarity conditions did not differ at Set Size 4, whereas the noveldistractors condition produced significantly longer RTs than the two familiar-distractors conditions at Set Sizes 10 and 16 (all $p \mathrm{~s}<.05$ ). No effects of set size or familiarity were observed for the low-similarity target-distractor pairs. Thus, when similarity between targets and distractors is low, the visual search is highly efficient regardless of target-distractor familiarity. When similarity is high, the search is efficient when distractors are familiar, regardless of target familiarity and inefficient when the distractors are novel.

The effect of target-distractor familiarity and feature similarity on error rates was similar to the effect on RTs. Error rates on target-present trials were higher when target-distractor the search is serial and easier when distractors are familiar regardless of target familiarity. Note that the stimuli are not drawn to scale.

similarity was high, $F(1,46)=81.33, p<.001, \eta_{\mathrm{p}}{ }^{2}=.64$, with a significant Similarity $\times$ Familiarity interaction, $F(2,92)=$ $19.38, p<.001, \eta_{\mathrm{p}}{ }^{2}=.30$. Similar to results for RTs, familiarity had a significant effect on error rates only when the targetdistractor similarity was high, with the novel-distractors condition resulting in higher error rates than the two familiardistractors conditions $(p \mathrm{~s}<.001)$, which themselves did not differ, $F(2,92)=29.36, p<.001, \eta_{\mathrm{p}}{ }^{2}=.39$.

For target-absent trials, RTs were generally faster in the familiar-distractors compared with novel-distractors conditions and when the distractors were dissimilar from the targets, main effects: $F(1,46)=265.61, p<.001, \eta_{\mathrm{p}}{ }^{2}=.85$, and $F(2$, $92)=40.67, p<.001, \eta_{\mathrm{p}}{ }^{2}=.47$, respectively. Distractor familiarity did not interact with target-distractor similarity. Naturally, given that no targets were present in these trials, all of the slopes reflected the serial nature of the search. Increases in set size resulted in corresponding increases in RT, $F(2,92)=121.87, p<.001, \eta_{\mathrm{p}}{ }^{2}=.73$, even more so in the high-similarity condition, $F(2,92)=19.39, p<.001, \eta_{\mathrm{p}}{ }^{2}=$ .30. Neither target-distractor similarity nor familiarity had an effect on error rates on target-absent trials, which were under $5 \%$ overall, but there was a small effect of set size, $F(2,92)=$ $4.66, p=.012, \eta_{\mathrm{p}}{ }^{2}=.09$, indicative of somewhat higher error rate at Set Size 4 than Set Size 16, $p=.017$.

Search-function slopes Table 3 displays search slopes for target-present and target-absent trials. For target-present trials, only the slopes in the three high-similarity conditions were significantly different from zero (all $p \mathrm{~s}<.05$ ). A two-way repeated-measures ANOVA confirmed a main effect of target-distractor similarity, $F(1,46)=39.96, p<.001, \eta_{\mathrm{p}}{ }^{2}=$ .47 , with a marginal effect of familiarity, $F(2,92)=2.39, p=$ $.097, \eta_{\mathrm{p}}{ }^{2}=.05$, but no interaction, $F(2,92)=2.23, p=.11, \eta_{\mathrm{p}}{ }^{2}$ $=.05$. To increase the power of the analysis, we collapsed the 


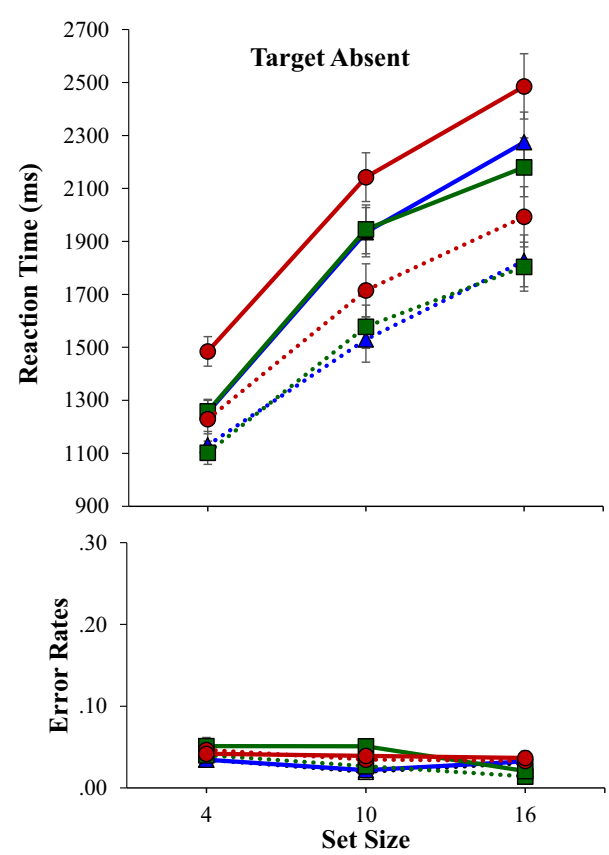

Fig. 3 Mean RTs and error rates as a function of target presence, similarity condition, familiarity condition, and set size for the target-

two low-similarity familiar-distractors conditions and the two high-similarity familiar-distractors conditions. The resulting two-way ANOVA revealed a main effect of familiarity $F(1$, 46) $=4.17, p=.047, \eta_{\mathrm{p}}{ }^{2}=.08$, and an interaction, $F(1,46)=$ $5.18, p=.028, \eta_{\mathrm{p}}^{2}=.10$. Follow-up Bonferroni-adjusted $t$ tests revealed a significant difference between slopes of familiar and novel distractor conditions when similarity was high, $t(46)=2.32, p=.025$, but not when it was low, $t(46)=.244, p$ $=.81$. With high similarity between targets and distractors, the slopes in the novel-distractors condition were nearly twice as steep as in the familiar-distractors conditions.

For target-absent trials, slopes were steeper in the highsimilarity $(M=81.7 \mathrm{~ms} /$ item $)$ compared with low-similarity conditions $(M=60.1 \mathrm{~ms} /$ item $), F(1,46)=27.59, p<.001, \eta_{\mathrm{p}}{ }^{2}=.38$, but did not differ across the three familiarity conditions, and there was no Similarity $\times$ Familiarity interaction $\left(F_{\mathrm{S}}<1\right)$. Finally, the slopes were significantly steeper in all conditions for target-absent, compared with target-present, trials (all $p s<.001$ ).

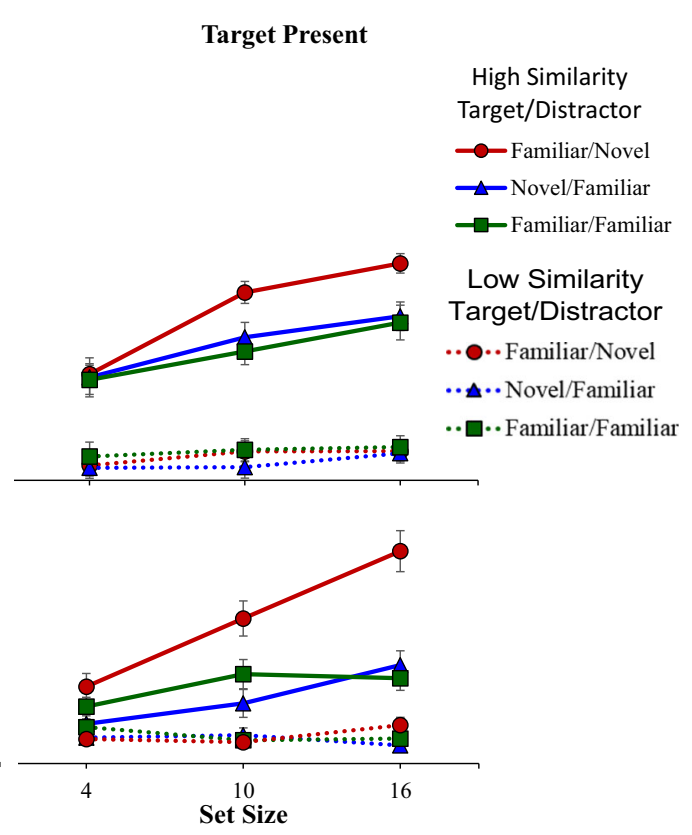

present and target-absent trials in Experiment 1 with error bars indicating standard errors. (Color figure online)

Comparison of slopes in target-present and target-absent trials We next examined differences between slopes of the two familiar-distractors conditions (collapsed) and the unfamiliardistractor condition as a function of target presence by conducting separate repeated-measures ANOVAs for highsimilarity and low-similarity conditions. This analysis should be particularly informative as a test of the three models shown in Fig. 1. When target-distractor similarity was high, both the main effects of target presence and of distractor familiarity were significant, $F(1,46)=67.98, p<.001, \eta_{\mathrm{p}}^{2}=.60$, and $F(1,46)=$ $4.52, p=.039, \eta_{\mathrm{p}}{ }^{2}=.09$, respectively, indicating steeper slopes in target-absent trials $(M=82.11 \mathrm{~ms} /$ item vs. $26.74 \mathrm{~ms} /$ item in target-present trials), as well as with novel $(M=59.11 \mathrm{~ms} /$ item $)$ compared with familiar distractors $(M=49.73 \mathrm{~ms} /$ item $)$. There interaction was only marginally significant, $F(1,46)=3.25, p=$ $.078, \eta_{\mathrm{p}}{ }^{2}=.066$, although the trend aligns with the results of analyses summarized earlier: That the slopes of conditions with novel versus familiar distractors differed only for target-present

Table 3 Search slopes $(\mathrm{ms} /$ item) and intercepts (estimated at midpoint) for the target-present and target-absent trials in Experiment 1

\begin{tabular}{|c|c|c|c|c|c|c|c|c|c|}
\hline \multirow{2}{*}{$\begin{array}{l}\text { Target-Distractor } \\
\text { Similarity }\end{array}$} & \multirow[t]{2}{*}{ Search Condition } & \multicolumn{2}{|c|}{ Target-Present Slope } & \multicolumn{2}{|c|}{ Target-Absent Slope } & \multicolumn{2}{|c|}{ Target-Present Intercept } & \multicolumn{2}{|c|}{ Target-Absent Intercept } \\
\hline & & Mean & $95 \% \mathrm{CI}$ & Mean & $95 \% \mathrm{CI}$ & Mean & $95 \% \mathrm{CI}$ & Mean & $95 \% \mathrm{CI}$ \\
\hline \multirow[t]{3}{*}{ Low } & Novel-Target Familiar-Distractor & 4.6 & {$[-0.4,9.6]$} & 58 & {$[56.1,69.9]$} & 966 & {$[894,1038]$} & 1496 & {$[1348,1643]$} \\
\hline & Familiar-Target Familiar-Distractor & 3 & {$[-3.8,9.8]$} & 58.5 & {$[46.8,70.3]$} & 1010 & {$[928,1092]$} & 1495 & {$[1358,1632]$} \\
\hline & Familiar-Target Novel-Distractor & 4.5 & {$[-0.3,9.2]$} & 63.7 & {$[47.6,79.8]$} & 992 & {$[921,1063]$} & 1646 & {$[1486,1806]$} \\
\hline \multirow[t]{3}{*}{ High } & Novel-Target Familiar-Distractor & 19.2 & {$[8.7,29.8]$} & 85 & {$[69.0,101.0]$} & 1416 & {$[1308,1524]$} & 1821 & {$[1666,1976]$} \\
\hline & Familiar-Target Familiar-Distractor & 18 & {$[7.8,28.3]$} & 76.7 & {$[60.9,92.6]$} & 1387 & {$[1290,1484]$} & 1795 & {$[1645,1945]$} \\
\hline & Familiar-Target Novel-Distractor & 34.8 & {$[23.6,46.0]$} & 83.4 & {$[68.4,98.3]$} & 1543 & {$[1428,1657]$} & 2038 & {$[1867,2208]$} \\
\hline
\end{tabular}


trials $(p=.025)$; they did not vary for target-absent trials $(p=$ $.561)$. Thus, the results of this analysis are more in line with Fig. 1c (i.e., distractor rejection speed plus other factors affect search efficiency) than with Fig. 1a (distractor rejection speed alone affects search efficiency) or Fig. $1 \mathrm{~b}$ (distractor rejection speed is not a factor in search efficiency).

When similarity was low, target-absent slopes were significantly steeper $(60.96 \mathrm{~ms} /$ item $)$ than target-present slopes (4.14 $\mathrm{ms} /$ item) were, $F(1,46)=88.64, p<.001, \eta_{\mathrm{p}}{ }^{2}=.66$, but neither the main effect of familiarity nor the interaction were statistically significant $(p s>.39)$. Thus, the distractor rejection rate was not affected by the familiarity of distractors, whether or not distractors and targets were highly similar or dissimilar.

Search-function intercepts Table 3 displays intercepts from linear fits to the individual RTs at a midpoint of the set size variable. For target-present trials, a two-way repeated-measures ANOVA revealed a main effect of target-distractor similarity, $F(1,46)=357.97, p<.001, \eta_{\mathrm{p}}{ }^{2}=.89$, and familiarity, $F(2,92)=11.87, p<.001, \eta_{\mathrm{p}}{ }^{2}=.21$, as well as an interaction $F(2,92)=11.94, p<.001, \eta_{\mathrm{p}}{ }^{2}=.21$. Follow-up Bonferroniadjusted comparisons showed no significant differences among any of the familiarity conditions when targetdistractor similarity was low (all $p \mathrm{~s}>.111$ ), as well as no difference between the two familiar-distractors conditions when similarity was high $(p=1.000)$. The intercepts in the familiar-target, novel-distractors condition, however, were $127-156 \mathrm{~ms}$ higher than the intercepts in the two familiardistractors conditions $(p<.001)$.

For target-absent trials, the intercepts in the low-similarity conditions were $400-500 \mathrm{~ms}$ less than the high-similarity conditions, $F(1,46)=265.6, p<.001, \eta_{\mathrm{p}}{ }^{2}=.85$. There was also a main effect of familiarity, $F(2,92)=40.67, p<.001, \eta_{\mathrm{p}}{ }^{2}=.47$, with intercepts in the two familiar-distractors conditions being almost $200 \mathrm{~ms}$ less than the novel-distractors condition (both $p \mathrm{~s}<.001$ ). There was no interaction, $F(2,92)=2.11, p=.127, \eta_{\mathrm{p}}{ }^{2}=.04$.

\section{Comparison of intercepts in target-present and target-absent} trials For high-similarity stimuli, a significant interaction between the presence of target and familiarity suggested that the distractor familiarity had a greater effect in target-absent trials $(230 \mathrm{~ms}, p<.001)$ than in target-present trials $(141 \mathrm{~ms}, p<$ $.001), F(1,46)=5.96, p=.018, \eta_{\mathrm{p}}{ }^{2}=.12$. For low-similarity stimuli, the intercept for the novel-distractors condition was $151 \mathrm{~ms}$ higher than the familiar-distractors conditions $(p<$ .001 ), whereas no such difference was observed with targetpresent trials $(p=.770), F(1,46)=17.60, p<.001, \eta_{\mathrm{p}}{ }^{2}=.28$.

\section{Discussion}

Our results show that, in accordance with previous findings (Malinowski \& Hübner, 2001; Mruczek \& Sheinberg, 2005; Shen \& Reingold, 2001; Wolfe, 2001), familiarity of the distractors, rather than the target, is responsible for the facilitation when searching for a novel target among familiar distractors versus a familiar target among novel distractors. However, distractor familiarity expedites the search only when there is a high degree of feature overlap between targets and distractors. With minimal overlap the difference in lowlevel stimulus features is salient, and familiarity does not influence search efficiency: The search slopes are essentially flat, regardless of familiarity, indicating that the target is detected preattentively. Conversely, when target-distractor similarity is high, the difference in basic features between the target and distractors precludes target pop-out and necessitates a more demanding attentive search. The novelty of background stimuli modulates the efficiency of this search.

Although looking for a familiar target among familiar distractors is as efficient as looking for a novel target among familiar distractors, we hesitate to conclude that the search asymmetry is solely due to a faster rejection speed elicited by familiar distractors. On the one hand, the steeper slopes produced by searching for a familiar target among novel distractors suggest a per-item processing cost to novel compared to familiar distractors - that is, a cost in search efficiency. In contrast, the search slopes in the homogeneous target-absent trials did not differ between the three familiarity conditions. While we are cautious about drawing conclusions on the basis of a null finding, the lack of a significant difference in target-absent slopes could indicate that processing costs per item remained constant across different familiarity conditions. If we were to assume that the slopes in the targetabsent trials genuinely reflect the speed of searching through the array of distractors (Woodman, Vogel, \& Luck, 2001), we would have to surmise that the speeds of rejecting familiar distractors and novel distractors are indeed comparable.

Another reason to doubt that distractor rejection speed is the main driving force behind the search asymmetry comes from the analyses of RTs/intercepts, which show a pronounced cost when searching through displays with novel distractors under conditions of high target-distractor similarity. These costs are not driven by distractor rejection speed; they reflect the influence of processes outside of item selection.

Finally, our results pinpoint several discrepancies in RTs of target-present and target-absent trials, which can be taken as further, albeit indirect, evidence that factors other than the slower rejection speed of novel distractors contribute to the search asymmetry. For instance, in the low-similarity condition, the target-distractor familiarity appears to play no role in modulating search efficiency when the target is present, but results in a 150 -ms cost for the familiar-target noveldistractors RTs on target-absent trials. In the high-similarity condition, the discrepancy between the two familiardistractors conditions and the unfamiliar-distractors condition is magnified for target-absent trials (by approximately $230 \mathrm{~ms}$ ) compared with target-present trials (by approximately 140 
ms). Finally, we found no significant differences in targetpresent RTs between the three high-similarity conditions at Set Size 4: Had the search asymmetry in our experiment been singularly due to faster rejection speed of familiar distractors, RTs for any of the three high-similarity conditions at Set Size 4 for the target-absent trials would have accorded with those for the target-present trials and thus would not have differed.

To sum up these observations, distractor familiarity influences search RTs in the absence of a highly distinctive target to capture attention. Search slopes, however, differ as a function of distractor familiarity only for target-present but not targetabsent trials, and only when target-distractor similarity is high, which activates a capacity-limited search. Therefore, we do not have sufficient evidence to surmise that the faster speed of rejecting familiar distractors is the reason for facilitated detection of a novel target among familiar distractors over a familiar target among novel distractors. Experiment 2 affords another opportunity to examine the role of distractor rejection speed and to rule out other possibilities for the lack of differences in slopes in the target-absent trials and the discrepancy in search times/intercepts between the target-present and target-absent trials. Since Experiment 1 utilized fairly large eccentricities (25 degrees horizontal, 10 degrees vertical) with stimuli appearing in random locations, it is possible that incidental clustering of stimuli may have contributed to the search time differential. Greater eccentricities are also associated with lower visual acuity and longer duration saccades (Mruczek \& Sheinberg, 2005), which will affect search latencies (Carrasco, Evert, Chang, \& Katz, 1995). Consequently, we wanted to get a cleared picture of what happens when the number of distractors is low and the visual field restricted. Thus, in Experiment 2 we employed much smaller eccentricities and arranged stimuli along the circumference of an imaginary circle. The range of set sizes was also reduced to enhance the resolution of responses at smaller set sizes.

\section{Experiment 2}

\section{Method}

Participants Sixty undergraduate students (ages 18-22 years) with normal or corrected-to-normal vision completed the study in exchange for course credit. Eight participants were excluded from analysis due to low (less than $86 \%$ ) response accuracy.

Stimuli and procedure Experiment 2 used the same 3 (targetdistractor familiarity) $\times 2$ (target-distractor similarity) design and stimuli as Experiment 1. Stimuli were presented in sets of $2,4,6$, and 8 , for a total of 480 trials and were arranged along the circumference of an imaginary circle with a diameter of $10^{\circ}$ of the visual angle. We also varied the duration of the fixation point between $500 \mathrm{~ms}$ and $1000 \mathrm{~ms}$. Participants were required to repeat blocks with low accuracy and were dismissed from the study if unable to maintain overall accuracy of $86 \%$ or greater. Other aspects of the procedure were comparable to those in Experiment 1.

\section{Results}

Response times and error rates The pattern of results in the current experiment was similar to those in Experiment 1. For the target-present trials (see Fig. 4), a three-way repeated-measures ANOVA showed that RTs were longer in the high, compared with low, similarity condition, $F(1,51)=147.78, p<$ $.001, \eta_{\mathrm{p}}{ }^{2}=.74$, as well as in the familiar-target/novel-distractor condition compared with the two familiar-distractors conditions $(p \mathrm{~s}<.001), F(2,102)=10.19, p<.001, \eta_{\mathrm{p}}^{2}=.17$. There was also a main effect of set size, $F(3,153)=12.32, p<.001, \eta_{\mathrm{p}}{ }^{2}=$ .20 , and a significant three-way interaction between similarity, familiarity, and set, $F(6,306)=2.80, p=.011, \eta_{\mathrm{p}}{ }^{2}=.05$.

Follow-up ANOVAs revealed that when the targetdistractor similarity was low, neither target-distractor familiarity nor set size manifested a main effect or interacted. When similarity was high, however, the novel-distractors condition had longer RTs than the two familiar-distractors conditions ( $p$ s $<.001)$. Furthermore, the RTs for the three conditions did not differ at Set Size $2(p=.199)$, but the novel-distractors condition RT was increasingly longer than the two familiardistractors conditions at Set Sizes 4, $F(2,102)=4.47, p=$ $.014, \eta_{\mathrm{p}}{ }^{2}=.08 ; 6, F(2,102)=4.79, p=.010, \eta_{\mathrm{p}}{ }^{2}=.09 ;$ and $8, F(2,102)=14.20, p<.001, \eta_{\mathrm{p}}{ }^{2}=.22$.

Similar to Experiment 1, errors were higher in the highsimilarity condition, $F(1,51)=44.11, p<.001, \eta_{\mathrm{p}}^{2}=.46$, although a significant Similarity $\times$ Familiarity interaction, $F(2$, $102)=8.88, p<.001$, revealed no effect of target-distractor familiarity when targets and distractors were dissimilar, but an increase in errors in the novel-distractors condition when they were similar $(p \leq .002$; the two familiar-distractors conditions did not differ significantly), $F(2,102)=11.77, p<.001, \eta_{\mathrm{p}}{ }^{2}=.19$.

For target-absent trials, a three-way repeated-measures ANOVA revealed a significant main effect of the targetdistractor similarity and familiarity on RT, $F(1,51)=$ $138.91, p<.001, \eta_{\mathrm{p}}{ }^{2}=.73$, and $F(2,102)=61.35, p<.001$, $\eta_{\mathrm{p}}{ }^{2}=.55$, respectively: RTs were longer in the high-similarity conditions $(p<.001)$, and novel distractors resulted in significantly longer RTs than familiar distractors $(p<.001)$. Followup analyses revealed that while the two familiar-distractors conditions did not differ, both produced significantly shorter RTs than the familiar-target/novel-distractors condition at almost every set size for both high-similarity and low-similarity pairings ( $p s \leq .028$ ). The only exceptions were set sizes 4 and 6: When target-distractor similarity was low, the familiar-target/familiar-distractors and the familiar-target/noveldistractors conditions did not differ $(p=.911$ at Set Size $4 ; p$ $=.118$ at Set Size 6). 

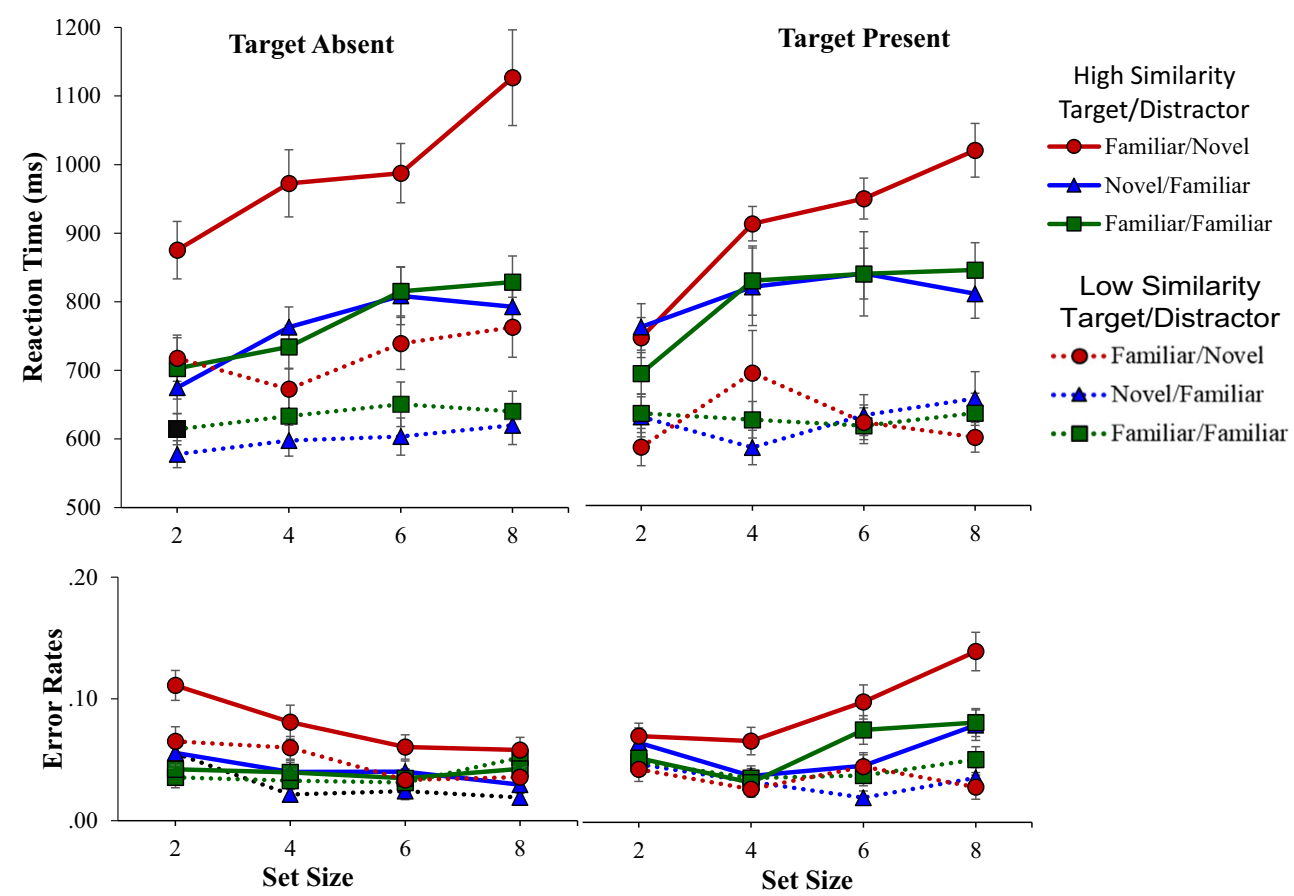

Fig. 4 Mean RTs and error rates as a function of target presence, similarity condition, familiarity condition, and set size for the target-absent and targetpresent trials in Experiment 2 with error bars indicating standard errors. (Color figure online)

With respect to errors, the high-similarity condition produced a significantly higher error rate than the low-similarity condition did, $F(1,51)=14.74, p<.001, \eta_{\mathrm{p}}{ }^{2}=.22$. Familiarity also had a significant main effect, $F(2,102)=$ $22.43, p<.001, \eta_{\mathrm{p}}{ }^{2}=.31$ : Error rate for the noveldistractors condition was significantly higher than the two familiar-distractors conditions $(p<.001)$.

Search-function slopes Table 4 displays search slopes for the six search conditions (2: similarity $\times 3$ : familiarity) for both the target-present and target-absent trials. A repeated measures ANOVA on target-present slopes revealed main effects of familiarity $F(2,102)=3.52, p=.033, \eta_{\mathrm{p}}{ }^{2}=.07$ and similarity $F(1,51)=32.81, p<.001, \eta_{\mathrm{p}}{ }^{2}=.39$, qualified by an interaction $F(2,102)=7.43, p=.001, \eta_{\mathrm{p}}^{2}=.13$. When the target-distractor similarity was low, search slopes were efficient $(<10 \mathrm{~ms} /$ item $)$ and did not differ across familiarity conditions. When similarity was high, the familiar-target/novel-distractors condition produced a slope five times steeper $(M=42.8 \mathrm{~ms} / \mathrm{item})$ than the novel-target/familiar-distractors condition $(8.2 \mathrm{~ms} /$ item; $p=$ $.001)$ and nearly twice as steep as the familiar-target/familiardistractors condition $(23.1 \mathrm{~ms} /$ item; $p=.059), F(2,102)=8.04$, $p=.001, \eta_{\mathrm{p}}{ }^{2}=.14$. The search slopes did not differ significantly between the familiar-target/familiar-distractors and novel-target/familiar-distractors conditions ( $p=.284$ ).

For the target-absent trials, slopes were steeper in the highsimilarity $(M=27.1 \mathrm{~ms} /$ item $)$ compared with low-similarity conditions $(M=7.2 \mathrm{~ms} /$ item $), F(1,51)=27.88, p<.001, \eta_{\mathrm{p}}{ }^{2}=$ .35 , and there was a marginally significant effect of familiarity,
$F(2,102)=2.98, p=.055, \eta_{\mathrm{p}}{ }^{2}=.06$, suggesting a somewhat steeper slope in the novel-distractors conditions $(M=24.27$ $\mathrm{ms} /$ item $)$ than in the familiar-distractors conditions $(M=13.57$ $\mathrm{ms} /$ item), but no interaction $(F<1)$. Notably, the target-absent slopes of the three low-similarity conditions were not different from zero, while the slopes in the three high-similarity conditions were all reliably above zero (see Table 4).

Comparison of slopes in target-present and target-absent trials Similarly to Experiment 1, we wanted to determine whether there were differences between slopes of the two familiardistractors conditions (collapsed) and the unfamiliar-distractor condition as a function of target presence. Repeated-measures ANOVAs were carried out separately for high-similarity and low-similarity conditions. When target-distractor similarity was high, only the main effect of distractor familiarity was significant, $F(1,51)=12.61, p=.001, \eta_{\mathrm{p}}^{2}=.20$, indicating steeper slopes with novel $(M=40.61 \mathrm{~ms} /$ item $)$ compared with familiar distractors $(M=18.55 \mathrm{~ms} /$ item $)$. When similarity was low, neither the two main effects nor the interaction were statistically significant (all $p \mathrm{~s}>.09$ ). Finally, a series of paired $t$ tests revealed that the target-absent slopes did not differ from target-present slopes in any of the experimental conditions (all $p \mathrm{~s}>.11)$.

Search-function intercepts Intercepts from linear fits to the individual RTs are shown in Table 4, centered on the set size variable. For target-present trials, a two-way repeated-measures ANOVA revealed a main effect of target-distractor 
Table 4 Search slopes (ms/item) and intercepts (estimated at midpoint) for the target-present and target-absent trials in Experiment 2

\begin{tabular}{|c|c|c|c|c|c|c|c|c|c|}
\hline \multirow{2}{*}{$\begin{array}{l}\text { Target-Distractor } \\
\text { Similarity }\end{array}$} & \multirow{2}{*}{ Search Condition } & \multicolumn{2}{|c|}{ Target-Present Slope } & \multicolumn{2}{|c|}{ Target-Absent Slope } & \multicolumn{2}{|c|}{ Target-Present Intercept } & \multicolumn{2}{|c|}{ Target-Absent Intercept } \\
\hline & & Mean & $95 \% \mathrm{CI}$ & Mean & $95 \% \mathrm{CI}$ & Mean & $95 \% \mathrm{CI}$ & Mean & $95 \% \mathrm{CI}$ \\
\hline \multirow[t]{3}{*}{ Low } & Novel-Target Familiar-Distractor & 6.4 & {$[-2.4,15.1]$} & 6.6 & {$[-1.6,14.9]$} & 625 & {$[572,678]$} & 600 & {$[562,637]$} \\
\hline & Familiar-Target Familiar-Distractor & -0.4 & {$[-8.1,7.3]$} & 4.8 & {$[-3.7,13.2]$} & 628 & {$[584,671]$} & 635 & {$[587,682]$} \\
\hline & Familiar-Target Novel-Distractor & -1.5 & {$[-9.8,6.8]$} & 10.1 & {$[-0.3,20.5]$} & 625 & {$[569,680]$} & 723 & {$[662,784]$} \\
\hline \multirow[t]{3}{*}{ High } & Novel-Target Familiar-Distractor & 8.2 & {$[-1.8,18.2]$} & 20 & {$[7.9,32.0]$} & 806 & {$[736,877]$} & 760 & {$[698,821]$} \\
\hline & Familiar-Target Familiar-Distractor & 23.1 & {$[11.6,34.6]$} & 22.9 & {$[9.2,36.7]$} & 800 & {$[721,879]$} & 770 & {$[709,832]$} \\
\hline & Familiar-Target Novel-Distractor & 42.8 & {$[27.5,58.1]$} & 38.5 & {$[24.3,52.6]$} & 904 & {$[814,996]$} & 991 & {$[899,1082]$} \\
\hline
\end{tabular}

similarity, $F(1,51)=147.78, p<.001, \eta_{\mathrm{p}}{ }^{2}=.74$, and familiarity, $F(2,102)=10.19, p<.001, \eta_{\mathrm{p}}{ }^{2}=.17$, as well as an interaction, $F(2,102)=9.45, p<.001, \eta_{\mathrm{p}}{ }^{2}=.16$. Follow-up Bonferroni-adjusted comparisons showed no significant differences among any of the familiarity conditions when targetdistractor similarity was low (all $p \mathrm{~s}=1.000$ ), as well as no difference between the two familiar-distractors conditions when similarity was high $(p=1.000)$. The intercepts in the familiar-target, novel-distractors condition, however, were approximately $100 \mathrm{~ms}$ higher than the intercepts in the two familiar-distractors conditions $(p<.001)$.

For target-absent trials, the intercepts in the low-similarity conditions were approximately $190 \mathrm{~ms}$ less than the highsimilarity conditions, $F(1,51)=138.91, p<.001, \eta_{\mathrm{p}}{ }^{2}=.73$. There was also a main effect of familiarity, $F(2,102)=61.35$, $p<.001, \eta_{\mathrm{p}}{ }^{2}=.55$, with intercepts in the two familiardistractors conditions being approximately $165 \mathrm{~ms}$ less than the novel-distractors condition (both $p \mathrm{~s}<.001$ ). The interaction was significant, $F(2,102)=9.45, p<.001, \eta_{\mathrm{p}}{ }^{2}=.16$ : With low similarity, the two familiar-distractors conditions yielded intercepts $105 \mathrm{~ms}$ lower than the novel-distractors condition. When similarity was high, the difference between the two familiar-distractors conditions and the noveldistractors condition was magnified: The two familiardistractors conditions produced intercepts approximately $225 \mathrm{~ms}$ lower than the novel-distractors condition (all post hoc Bonferroni $p$ values were significantly different, $p<$ .001 , except for the differences between the novel-target familiar-distractors and familiar-target familiar distractors conditions, $p s>.05)$.

\section{Comparison of intercepts in target-present and target-absent} trials We collapsed the midpoint intercepts of the two familiardistractor conditions and compared them to the intercepts of the novel-distractor condition across target-present and targetabsent trials. A repeated-measures ANOVA revealed a main effect of distractor familiarity, $F(1,51)=67.22, p<.001, \eta_{\mathrm{p}}{ }^{2}=$ .57 , qualified by a significant interaction: While novel distractors consistently produced higher intercepts, post hoc Bonferroni comparisons revealed that the difference between the novel and familiar distractors for target-absent trials was considerably greater $(M=255 \mathrm{~ms}, p<.001)$ than the difference for target-present trials $(M=102 \mathrm{~ms}, p<.001)$.

\section{Discussion}

Experiment 2 replicated the key finding of Experiment 1: For target-present trials, searching among familiar distractors was more efficient than among novel distractors regardless of target familiarity, but only when the similarity between the target and distractors was high. When the target and distractors were dissimilar, the asymmetry disappeared, and the search function slopes reflected an efficient search during which the target popped out regardless of target or distractor familiarity.

Furthermore, consistent with the results of Experiment 1, the search-function intercepts carried much of the cost of distractor novelty; these costs were apparent both for targetpresent and target-absent, homogeneous, search displays. Although at first glance, the considerably steeper slope of locating the familiar target among novel distractors in comparison to the familiar-distractors conditions appears to suggest a difference in distractor rejection speed, the rate of searching through the homogeneous distractors did not seem to vary with their familiarity.

This outcome is inconsistent with the conclusion that the speed of processing and rejecting familiar distractors is indeed faster than the speed of processing and rejecting unfamiliar distractors. The findings of Experiment 2, therefore, align best with the model drawn in Panel $\mathrm{C}$ of Figure 1, in which distractor familiarity affects processes outside item selection. They also confirm our supposition that the lack of a difference in the distractor rejection speed and the discrepancy between target-present and target-absent trials cannot be reduced to factors concerning stimulus presentation.

\section{General discussion}

Our research addresses the basic question of how the attentional system prioritizes visual search by demonstrating that 
the observer's familiarity with the background information becomes increasingly important to search efficiency when low-level features that differentiate objects (targets) from the background (distractors) increase in the degree of overlap. Specifically, we aimed to clarify the mechanisms that could explain why searching for a novel target among familiar distractors is easier than searching for a familiar target among novel distractors. The explanations for this search asymmetry have not been consistent, with some authors suggesting that attention can be captured preattentively by a novel target (Treisman \& Gormican, 1988) or that attentional capture requires a difference in familiarity between targets and distractors (Wang et al., 1994). Others argued that the familiarity of distractors facilitates their rejection in the capacitylimited attentive stage of visual search (Malinowski \& Hübner, 2001; Mruczek \& Sheinberg, 2005; Shen \& Reingold, 2001). These explanations, however, fall short of accounting for the results of our experiments, which carefully controlled the number of basic features shared by targets and distractors and attempted to minimize the role of target knowledge by using a search task without a predefined target.

The findings we report advance our understanding of the factors that give rise to the visual search asymmetries in several ways. First, in both experiments the data from the targetpresent trials concur with the previous findings that distractor, rather than target, familiarity drives the asymmetry: The search among familiar distractors was easier than among novel distractors regardless of target familiarity. However, this conclusion must be qualified by three observations. First, only when targets and distractors were highly similar was the search affected by distractor familiarity. Second, familiarity of distractors affects processes that lie outside of the capacity-limited item selection stage apart from the effects it might have on the distractor rejection rate. While the searchfunction slopes of locating a target among novel distractors were consistently higher than the slopes of searching for a target among familiar distractors, by analyzing the slopes of target-absent trials we found no differences in the rejection speed of familiar and novel distractors. On the contrary, different familiarity conditions produced similar slopes with respect to target-absent trials in both experiments. Thus, our results raise the previously-unexplored possibility that the speeds of rejecting familiar and novel distractors may indeed be comparable, in which case we must search for causes of the familiarity-driven asymmetry outside of the item selection stage. Our findings suggest that these causes may involve pre- or post-attentive factors such as search initiation, monitoring, and termination processes; these possibility are worthy of further examination. Third, if the speed of rejecting novel and familiar distractors indeed does not differ, given that the search asymmetry nonetheless occurred (i.e., the targetpresent slopes were steeper in the novel-distractors compared to the familiar-distractors conditions), it follows that distractor familiarity does not seem to be the sole factor contributing to the search asymmetry.

Our claim that distractor rejection speed does not fully explain the search asymmetry holds up regardless of search task parameters, such as stimulus eccentricity and set size. For instance, in Experiment 1 the target-absent slopes were considerably steeper than target-present slopes yet remained largely invariant between familiar and novel distractors (Table 3). A reduction in eccentricity and set size in Experiment 2 reduced the differences in slopes of targetpresent and target-absent trials, yet distractor familiarity did not affect the target-absent slopes (Table 4). Moreover, targetpresent slopes in both experiments were affected by distractor familiarity in a similar fashion: Steeper slopes were observed in the novel-distractors condition, whereas shallower slopes were found in the two familiar-distractors conditions. Primarily, however, our results indicate that the bulk of the differences between conditions with familiar distractors and novel distractors was carried by the intercept of the search function: In target-absent trials of both experiments, RT increments were additive with set size, indicating a fixed cost to encountering novel distractors during a search. In targetpresent trials, the intercept differences were found only when distractors and targets were orthographically similar; when the low-level features were distinct, the intercepts, like the slopes, remained invariant.

The degree of similarity between targets and distractors proved to be a powerful moderator of the discrepancy in target-present search slopes: With high similarity, the slopes were steeper when distractors were novel, suggesting greater per-item inspection times. However, the slopes did not vary with distractor familiarity when similarity between the target and distractors was low. In fact, low-similarity slopes in all conditions of our two experiments were under $6.5 \mathrm{~ms} /$ item, an indication of target "pop-out" that is characteristic of parallel search, much like what happens when we readily detect a single red circle among blue circles or a single vertical line among many horizontals. Thus, we must conclude that the familiarity of stimuli, be it targets or distractors, is not a factor in attentional selection when low-level features are sufficient to capture attention without the item-by-item search. On the other hand, when the low-level features of targets and distractors are similar enough to preclude a pop-out, familiarity of the distractors gains prominence in facilitating search efficiency as the difficulty of the search increases.

\section{The roles of distractor familiarity and target-distractor similarity in producing a search asymmetry}

Our main finding can be summed up by the following: When the target-distractor similarity is high, novel distractors produce steeper target-present slopes and higher intercepts, while 
in target-absent trials, only the intercepts, but not slopes, diverge. These results approximate the linear search functions displayed in Fig. 1c. When the target-distractor similarity is low, the convergence of target-present search-function slopes and intercepts indicates that the target pop-out eliminates any potential effects due to distractor familiarity. For target-absent trials, a difference in search intercepts, but not slopes, is consistent with the idea, depicted in Fig. 1b-c, that distractor familiarity does not necessarily affect distractor processing, but rather seems to engage preattentive or postattentive mechanisms. We will next discuss several potential explanations for this this pattern of results.

The presence of the search asymmetry, coupled with the observation of comparable target-absent slopes between the novel-distractors and familiar-distractors conditions, is at odds with previous explanations for the search asymmetry, such as the increase in the number of distractors that can be simultaneously rejected if they are familiar (Greene \& Rayner, 2001; Mruczek \& Sheinberg, 2005). A difference in search RTs (captured by search-function intercepts), but not search efficiency (captured by search-function slopes), suggests that distractor familiarity may affect mechanisms outside of stimulus selection (Wolfe, Oliva, Horowitz, Butcher, \& Bompas, 2012). Indeed, models of attentional selection, such as the guided search model (Wolfe, 1994), have shown that the efficiency of search through an array of distractors does not improve even after prolonged exposure to said distractors in extensive trials. Thus, rather than reducing the item selection rate, distractor familiarity may influence preattentive or postattentive processing, such as search monitoring and termination processes (e.g., Hout \& Goldinger, 2012; Visalli \& Vallesi, 2018).

For instance, when the distractors are unfamiliar, the decision to terminate the search may come later than when they are familiar. According to Chun and Wolfe (1996), the decision to terminate the search is set automatically based on a threshold that updates performance goals based on desired speed, accuracy, and a certain tolerance for guessing. Task parameters, such as display eccentricity, will further alter the decision threshold. The novelty of distractors may therefore delay the decision to terminate the search based on one or more of these criteria. On the other hand, familiar distractors may lower the observer's decision threshold regarding the presence of a singleton in a particular display or increase decision confidence (e.g., Kunar, Flusberg, Horowitz, \& Wolfe, 2007).

A search termination threshold can also be set adaptively depending on task parameters. Imagine that an observer encounters a trial with stimuli (primarily distractors) that, on prior trials, were associated with a confusable target (i.e., a target that's physically similar to distractors). Even as observers cannot increase the speed of the search through, and rejection of, distractors, they can implicitly establish a more conservative decision criterion whereby they might search longer through the distractor display before concluding that there is no unique item present. Thus, distractor familiarity might be considered a form of contextual cueing in which knowledge of stimulus properties truncates the search for a target by reducing the time to arrive at a conclusion. As such, familiarity effects should be apparent both on target-present and target-absent trials, as was indeed the case in our experiments. This reasoning can also explain why any familiarity effects are magnified with highly similar targets and distractors: Since we employed the same set of stimuli for both target-present and target-absent trials, and given that the target and the distractors in the target-present trials could be highly confusable, participants could have been more cautious about terminating the search in the high-similarity conditions if they suspected that a highly confusable target might also be present. In other words, there might be some practice effect such that the participants learned to associate characters from the high-similarity conditions with the potential presence of a highly confusable target, yielding longer RTs.

Another explanation for the RT advantage of familiar distractors concerns their effects on visual working memory (VWM), which provides temporary storage to a limited number of objects. Examination of our results (particularly Figs. 3 and 4) suggests that the costs of distractor novelty emerge with larger set sizes: For high-similarity targets, there are no costs at Set Size 4 in Experiment 1 or Set Size 2 in Experiment 2; for low-similarity targets, these costs are completely abolished because the target pops out of the display without the need for attentive search. These trends are consistent with a model that postulates a limit of three to four on the number of objects that can be held in VWM concurrently (Luck, 2008; Luck \& Vogel, 1997). According to Woodman et al. (2001), taxing the VWM capacity increases the intercepts, but not slopes, of search RTs (see also Wolfe et al., 2012). Therefore, holding novel distractors in working memory may require more processing resources that holding the familiar distractors would. The reasons for this outcome are unclear, but one possibility includes a more efficient perceptual grouping, or chunking, of familiar distractors. This reasoning is particularly fitting when we examine the data from our target-absent trials: When similarity is high, novel distractors add a constant per-item cost to the search process; when similarity is low, the cost is still fixed, but lower. This pattern of results suggests, yet again, that faster search times do not result from an increased ability to process individual items, but from other factors such as, potentially, altered decision thresholds, response confidence, or even a reduction in fixations per trial (e.g., Hout \& Goldinger, 2012).

Given that targets that share features with distractors will have a particular difficulty accessing VWM (Duncan \& Humphreys, 1989), we would expect a greater bottleneck in the storage of targets and distractors with highly similar features. Indeed, in both of our experiments, larger intercept and 
slope differences were obtained when similarity between targets and distractors was high. Adding to the perceptual load in the high-similarity condition is the number of features that comprised the high-similarity stimuli: In applying Fiset et al.' (2009) feature matrix to calculate the average number of unique features in distractors used in our study, we determined that the distractors in the high-similarity conditions had, on average, 10.5 features compared with 8.4 in the lowsimilarity conditions. Hence, the number of features that must be processed might increase the difficulty of the highsimilarity search: That factor alone will magnify search slopes by interfering with the visual system's ability to segment visual space if the oddball stimulus does not pop out. Conversely, when the perceptual load is low, in the language of the guided search theory of visual search (Wolfe, 1994) preattentive processes would enable the user to both identify the locations of potential targets and their identity, since one or more features of the low-similarity target is an odd-man out. Our results demonstrate that this preattentive capture happens regardless of target or distractor familiarity. When similarity between targets and distractors is high, however, preattentive processes are not sufficient to detect the oddball target, and identification must proceed during a limited-capacity stage of processing where the display is searched serially until the target is located. In that case, the search function slopes are significantly higher than zero.

Distractor novelty introduces a further load into the already-taxed attentive stage. The effects of this load can be manifested as increased search function slopes or intercepts. Our observations suggest that increasing the set size does not necessarily add per-item costs to the search RTs. Rather, the costs imposed by distractor familiarity appear to be fixed as the speed of distractor rejection remains constant regardless of set size. While this conclusion is plausible, given our data, it is limited by its nature as a "null result," albeit one that's clearly observed in both of the current experiments. On the other hand, if this reasoning is accurate, how could one explain the steeper slopes when searching for a target among novel, compared with familiar, distractors? We next consider whether these steeper slopes might occur because of the properties of the target.

\section{Do target characteristics enhance perceptual processing?}

In spite of the importance of distractors to search asymmetry, the role of the target cannot be discounted completely. Our results are consistent with the claim that the discrepancy between the responses on target-present and target-absent trials results from an increase in the general task completing efficiency (decrease in reaction times) when a familiar target is added to otherwise novel distractors. However, the advantage given by the familiar target may only occur under one of the two circumstances: Either when the set size is small or when the target-distractor similarity is low. What both circumstances share is that the perceptual load they demand from participants is relatively low. This is consistent with the perceptual load theory of selective attention (Lavie, 2005; Lavie \& Cox, 1997), according to which perception can process all stimuli automatically in a parallel fashion, but has limited capacity. As a result, task-irrelevant information can be processed when the perceptual load is low, and spare capacity can be used to process additional information. In contrast, when the task is demanding so that it fully exploits perceptual capacity, additional information cannot be processed. Hence, we speculate that when the perceptual load required by the task is low, additional information regarding target familiarity can be used to facilitate identifying the target. In contrast, when the perceptual load is high, as in those trials that had a large set size or high similarity between the target and distractors, target familiarity can no longer be utilized while distractor familiarity gains prominence in governing search efficiency.

It is also possible that target detection can be enhanced indirectly by distractor familiarity: Evidence suggests that an increase in the efficiency of perceptual grouping of familiar distractors can enhance the salience of a target (Yang, Chen, \& Zelinsky, 2009). Finally, the mere presence of the target might be sufficient to alter how distractors are processed. The discrepancy in target-present and target-absent slopes evident in our results does not preclude the possibility that the presence of the target modulates the distractor rejection rate.

\section{Conclusions and limitations}

Our findings are consistent with previous assertions (e.g., Duncan \& Humphreys, 1989; see also Wolfe, 2001) that efficient target detection is contingent upon the similarity of features between the target and the distractors: The target pops out of the array when that similarity is low-that is, when the target possesses distinguishing features. In those cases, distractors are not scrutinized, particularly when they are homogeneous. Increasing similarity between the target and the distractors necessitates the more demanding serial search, which leads to increases in RT with set size as well as a reduction in search efficiency. The novelty of distractors imposes global costs on the search performance outside of the attentive search, and the distractors' role becomes more prominent as perceptual load increases. Indeed, single-cell recordings support the view that more similar distractors are processed longer by the visual system than less similar distractors after evidence about both has been allowed to accumulate (Purcell et al., 2010).

Importantly, previous research has tended to focus on distractor processing speed and overlooked the effects of familiarity on other general processes like search monitoring 
and termination, which may play a prominent role in giving rise to the search asymmetry. Examination of the role of topdown strategic and experience-driven processes more broadly may provide a fruitful avenue for future research. While we argued for a key role of the search termination processes in driving the familiar-distractors search advantage, we must also consider the possibility that early perceptual processing has contributed to our results, particularly the effects due to target-distractor similarity. Buetti et al. (2016) demonstrated that processing of stimulus displays prior to when individual items are scrutinized can be affected by the target-distractor similarity: The visual system imposes elevated preattentive decision thresholds when stimulus similarity increases. These thresholds are global in nature, rather than affecting separate feature channels, and they increase search RTs. Our observation of elevated intercepts for high-similarity compared with low-similarity homogeneous (i.e., target-absent) displays is consistent with that view, although additional research is needed to tease apart the contribution of preattentive and postattentive processes to search asymmetries. More generally, the interaction between preattentive and postattentive processes can reveal more fully the effects of familiarity on visual search. For instance, eye tracking work suggests that during a search, after the observer's gaze locates the target preattentively, it wanders away before returning to the target twice as often in the familiar-target/novel-distractors than the novel-target/familiar-distractors trials (Zhaoping \& Frith, 2011).

One potential criticism of the current experiments concerns the external validity of results: Real-world search behaviors involve complex stimuli and substantial heterogeneity in distractors. In those situations, familiarity of the target may exert a stronger influence on the visual search than the familiarity of distractors (Qin et al. 2014). Similarly, certain complex targets, such as angry faces, may capture attention regardless of distractor familiarity or valence (Shasteen, Sasson, \& Pinkham, 2014). Finally, intermixing trials with search targets that were distractors in previous trials, as was done in our experiments, increases the difficulty of the visual search (Shiffrin \& Schneider, 1977). In spite of these limitations, understanding the principles of attentional capture can have a variety of real-life applications: For instance, impairments in attentional guidance, and particularly reduced sensitivity to stimulus novelty, can contribute to working memory impairments in clinical populations such as individuals with schizophrenia (Mayer, Kim, \& Park, 2014). Observations that familiar brand logos are detected faster than unfamiliar logos can improve our understanding of effective marketing (Qin et al., 2014), while understanding factors that distinguish objects from the background of the environment has broad applicability to reconnaissance, surveillance, and other military applications (Fincannon, Keebler, Jentsch, \& Curtis, 2013).
To conclude, our findings confirm the claim that the properties of distractors are the primary contributor to the more efficient identification of a novel target among familiar distractors than a familiar target among novel distractors. However, the absence of any difference in slopes in targetabsent trials casts doubt on the hypothesis that search asymmetry results solely from faster rejection speed of familiar distractors. Consequently, we speculate that distractor familiarity influences processing stages outside of attentional selection: This assertion is confirmed by our observation of consistently higher RTs when distractors are novel regardless of target presence. Yet given that the differences in slopes between the different familiarity conditions were nonetheless observed in target-present trials, we argue that target characteristics, or at least target presence, may also contribute to search asymmetry. While distractor familiarity acts primarily on preattentive or postattentive processes, the presence of the target may be the critical factor to facilitate the familiar distractor rejection rate. This outcome presents an intriguing possibility to be examined in future research. In fact, neuroimaging evidence indicates that familiar distractors can be suppressed during visual search at the same time as target selection is enhanced (Dent, Allen, Braithwaite, \& Humphreys, 2012; Seidl, Peelen, \& Kastner, 2012), and that distractor suppression can become difficult when distractors share features with targets. However, the careful controls afforded by manipulating the basic features of targets and distractors suggest that the similarity of these features is a key variable that affects the perceptual load on the visual system and determines the costs of the search and whether or not a search asymmetry will be observed. Broadly speaking, our results indicate that the study of search asymmetries continues to provide fertile grounds for testing the predictions of the many theories of attention, just as it did in the seminar work of Treisman and colleagues (Treisman \& Gormican, 1988; Treisman \& Souther, 1985).

Author note We thank Ken Sobel and an anonymous reviewer for helpful comments on an earlier version of the manuscript.

Open practices statement The data and materials for all experiments can be obtained from the corresponding author. None of the experiments were preregistered.

\section{References}

Awh, E., Belopolsky, A. V., \& Theeuwes, J. (2012). Top-down versus bottom-up attentional control: A failed theoretical dichotomy. Trends in Cognitive Sciences, 16(8), 437-443. https://doi.org/10. 1016/j.tics.2012.06.010

Buetti, S., Cronin, D. A., Madison, A. M., Wang, Z., \& Lleras, A. (2016). Towards a better understanding of parallel visual processing in 
human vision? Evidence for exhaustive analysis of visual information. Journal of Experimental Psychology: General, 145, 672-707. https://doi.org/10.1037/xge0000163

Carrasco, M., Evert, D. L., Chang, I., \& Katz, S. M. (1995). The eccentricity effect: Target eccentricity affects performance on conjunction searches. Perception \& Psychophysics, 57, 1241-1261.

Chun, M. M., \& Wolfe, J. M. (1996). Just say no: How are visual searches terminated when there is no target present? Cognitive Psychology, 30, 39-78.

Dent, K., Allen, H. A., Braithwaite, J. J., \& Humphreys, G. W. (2012). Parallel distractor rejection as a binding mechanism in search. Frontiers in Psychology, 3, 278. https://doi.org/10.3389/fpsyg. 2012.00278

Duncan, J., \& Humphreys, G. W. (1989). Visual search and stimulus similarity. Psychological Review, 96, 433-458. https://doi.org/10. 1037/0033-295X.96.3.433

Fincannon, T., Keepber, J. R., Jentsch, F., \& Curtis, M. (2013). The influence of camouflage, obstruction, familiarity and spatial ability on target identification from an unmanned ground vehicle. Ergonomics, 56(5), 739-751. https://doi.org/10.1080/00140139. 2013.771218

Fiset, D., Blais, C., Arguin, M., Tadros, K., Éthier-Majcher, C., Bub, D., \& Gosselin, F. (2009). The spatio-temporal dynamics of visual letter recognition. Cognitive Neuropsychology, 26, 23-35.

Geyer, L. H., \& DeWald, C. G. (1973). Feature lists and confusion matrices. Perception \& Psychophysics, 14, 471-482.

Greene, H. H., \& Rayner, K. (2001). Eye movements and familiarity effects in visual search. Vision Research, 41, 3763-3773.

Horstmann, G. (2009). Visual search for affective faces: Stability and variability of search slopes with different instances? Cognition and Emotion, 23, 355-379.

Horstmann, G., Becker, S. I., Bergmann, S. I., \& Burghaus, L. (2010). A reversal of the search asymmetry favouring negative schematic faces. Visual Cognition, 18, 981-1016.

Hout, M. C., \& Goldinger, S. D. (2012). Incidental learning speeds visual search by lowering response thresholds, not by improving efficiency: Evidence from eye movements. Journal of Experimental Psychology: Human Perception \& Performance, 38(1), 90-112. https://doi.org/10.1037/a0023894

Kunar, M. A., Flusberg, S., Horowitz, T. S., \& Wolfe, J. M. (2007). Does contextual cuing guide the deployment of attention? Journal of Experimental Psychology: Human Perception \& Performance, 33, $816-828$

Lavie, N. (2005). Distracted and confused?: Selective attention under load. Trends in Cognitive Sciences, 9, 75-82.

Lavie, N., \& Cox, S. (1997). On the efficiency of visual selective attention: Efficient visual search leads to inefficient distractor rejection. Psychological Science, 8, 395-398.

Lee, K. R., Sobel, K. V., York, A. K., \& Puri, A. M. (2018). Dissociating parallel and serial processing of numerical value. Journal of Numerical Cognition, 4(2), 360-379. https://doi.org/10.5964/jnc. v4i2.133

Luck, S. J., \& Vogel, E. K. (1997). The capacity of visual working memory for features and conjunctions. Nature, 390, 279-281.

Luck, S. J. (2008). Visual short-term memory. In S. J. Luck \& A. Hollingworth (Eds.), Visual memory (pp. 43-85). New York, NY: Oxford University Press.

Malinowski, P., \& Hübner, R. (2001). The effect of familiarity on visualsearch performance: Evidence for learned basic features. Perception \& Psychophysics, 63, 458-463.

Mruczek, R. B., \& Sheinberg, D. L. (2005). Distractor familiarity leads to more efficient visual search for complex stimuli. Perception \& Psychophysics, 67, 1016-1031.

Navalpakkam, V., \& Itti, L. (2005). Modeling the influence of task on attention. Vision Research, 45, 205-231.
Purcell, B. A., Heitz, R. P., Cohen, J. Y., Schall, J. D., Logan, G. D., \& Palmeri, T. J. (2010). Neurally constrained modeling of perceptual decision making. Psychological Review, 117, 1113-1143.

Qin, X. A., Koutstaal, W., \& Engel, S. A. (2014). The hard-won benefits of familiarity in visual search: Naturally familiar brand logos are found faster. Attention, Perception, \& Psychophysics, 76, 914 930. https://doi.org/10.3758/APP.72.5.1267

Palmer, J., Verghese, P., \& Pavel, M. (2000). The psychophysics of visual search. Vision Research, 40, 1227-1268.

Rauschenberger, R., \& Chu, H. (2006). The effects of stimulus rotation and familiarity in visual search. Perception \& Psychophysics, 68(5), 770-775.

Reicher, G. M., Snyder, C. R. R., \& Richards, J. T. (1976). Familiarity of background characters in visual scanning. Journal of Experimental Psychology: Human Perception \& Performance, 2, 522-530.

Rosenholtz, R. (2001). Search asymmetries? What search asymmetries? Perception \& Psychophysics, 63, 476-489.

Rosenholtz, R., Huang, J., Raj, A., Balas, B. J., \& Ilie, L. (2012). A summary statistic representation in peripheral vision explains visual search. Journal of Vision, 12(4), 1-17. https://doi.org/10.1167/12.4. 14

Saiki, J. (2008). Stimulus-driven mechanisms underlying visual search asymmetry revealed by classification image analyses. Journal of Vision, 8(4), 30, 1-19.

Saiki, J., Koike, T., Takahashi, K., \& Inoue, T. (2005). Visual search asymmetry with uncertain targets. Journal of Experimental Psychology: Human Perception and Performance, 31(6), 12741287.

Seidl, K. N., Peelen, M. V., \& Kastner, S. (2012). Neural evidence for distracter suppression during visual search in real-world scenes. The Journal of Neuroscience, 32(34), 11812-11819.

Shasteen, J. R., Sasson, N. J., \& Pinkham, A. E. (2014). Eye tracking the face in the crowd task: Why are angry faces found more quickly? PLOS ONE, 9(4), e93914. https://doi.org/10.1371/journal.pone. 0093914

Shen, J., \& Reingold, E. M. (2001). Visual search asymmetry: The influence of stimulus familiarity and low-level features. Perception \& Psychophysics, 63, 464-475.

Shiffrin, R. M., \& Schneider, W. (1977). Controlled and automatic human information processing: II. Perceptual learning, automatic attending and a general theory. Psychological Review, 84, 127-190.

Treisman, A. M., \& Gelade, G. (1980). A feature-integration theory of attention. Cognitive Psychology, 12, 97-136. https://doi.org/10. 1016/0010-0285(80)90005-5

Treisman, A., \& Gormican, S. (1988). Feature analysis in early vision: Evidence from search asymmetries. Psychological Review, 95, 1548

Treisman, A., \& Souther, J. (1985). Search asymmetry: A diagnostic for preattentive processing of separable features. Journal of Experimental Psychology: General, 114, 285-310.

Visalli, A., \& Vallesi, A. (2018). Monitoring processes in visual search enhanced by professional experience: The case of orange qualitycontrol workers. Frontiers in Psychology, 9, 145. https://doi.org/10. 3389/fpsyg.2018.00145

Wang, Q., Cavanagh, P., \& Green, M. (1994). Familiarity and pop-out in visual search. Perception \& Psychophysics, 56, 495-500.

Wang, L., Zhang, K., He, S., \& Jiang, Y. (2010). Searching for life motion signals: Visual search asymmetry in local but not global biologicalmotion processing. Psychological Science, 21, 1083-1089.

Wolfe, J. M. (1998). What do 1,000,000 trials tell us about visual search? Psychological Science, 9, 33-39.

Wolfe, J. M. (2001). Asymmetries in visual search: An introduction. Perception \& Psychophysics, 63, 381-389.

Wolfe, J. M., Cave, K. R., \& Franzel, S. L. (1989). Guided search: An alternative to the feature integration model for visual search. Journal 
of Experimental Psychology: Human Perception \& Performance, $15,419-433$.

Wolfe, J. M., Oliva, A., Horowitz, T. S., Butcher, S. J., \& Bompas, A. (2012). Segmentation of objects from backgrounds in visual search tasks. Vision Research, 42, 2985-3004.

Woodman, G. F., Vogel, E. K., \& Luck, S. J. (2001). Visual search remains efficient when visual working memory is full. Psychological Science, 12, 219-224.

Yang, H., Chen, X., \& Zelinsky, G. J. (2009). A new look at novelty effects: Guiding search away from old distractors. Attention, Perception, \& Psychophysics, 71, 554-564.
Zhaoping, L., \& Frith, U. (2011). A clash of bottom-up and top-down processes in visual search: The reversed letter effect revisited. Journal of Experimental Psychology: Human Perception and Performance, 37(4), 997-1006.

Publisher's note Springer Nature remains neutral with regard to jurisdictional claims in published maps and institutional affiliations. 